\title{
Cosmic ray composition at the ankle in the energy spectrum
}

\author{
T. Wibig ${ }^{1}$ and A. W. Wolfendale ${ }^{2}$ \\ ${ }^{1}$ Physics Dept., University of Łódź; Sołtan Inst. Nucl. Studies, Łódź, Poland \\ ${ }^{2}$ Department of Physics, Durham University, Durham, UK
}

Received: 6 November 2010 - Revised: 3 March 2011 - Accepted: 4 March 2011 - Published: 13 July 2011

\begin{abstract}
Recent results from the Pierre Auger Observatory and the Hi-Res array have been analysed hoping to derive a strong statement about the mass composition in the region of the ankle, where the change from Galactic to ExtraGalactic cosmic ray flux should take place, at least according to some theoretical predictions. It is shown that there are problems in reconciling the data with expectation.
\end{abstract}

\section{Introduction}

The question about the nature of the particles of energies around the spectral feature called the "ankle", the sharp change of slope around $3 \mathrm{EeV}$, is one of the most important and still open questions in the Ultra High-Energy Cosmic Ray (UHECR) domain. The lower energy cosmic rays are known to be mainly confined within the Galaxy, and it is known that the Galactic field is too weak to contain particles of energies above $\simeq 10^{19} \mathrm{eV}$. It is believed by us and others that the ankle is related to the extragalactic (EG) origin of the cosmic ray particles.

It must be stated, however, that Berezinsky et al. (2006) consider that the ankle is an intrinsic property of EG particles. In the present work, however, we persist with our view that the ankle is at the cross-over point for $\mathrm{G}$ and $\mathrm{EG}$ particles largely in view of its' sharpness.

The results published (Abraham et al., 2010; Sokolsky and Thompson, 2007; Abbasi et al., 2010) consist of high statistics energy spectrum data, the average position of the shower maximum $\left(x_{\max }\right)$ and the spread of this maximum as a function of energy in the range from $10^{17} \mathrm{eV}$ to $3 \times 10^{19} \mathrm{eV}$. We would like to use them to obtain information about the mass composition in the "ankle" region.

Correspondence to: $\mathrm{T}$. Wibig

(wibig@zpk.u.lodz.pl)

\section{The model of the "ankle"}

We have assumed the model proposed some time ago by Szabelski et al. (2002) i.e. the G, EG transition.

The analysis in Wibig and Wolfendale (2005) of the shape of CR spectra measured for energies above $10^{17} \mathrm{eV}$ by different Extensive Air Shower (EAS) arrays has been augmented by recent data from PAO and Hi-Res just referred to. The experiments confirm the universal shape of the ankle which can be read as a sum of the EG power-law component with an index of -2.4 with a cut related to GZK or photodisintegration (or some other processes involving the source energy limits) which is above our present interest $\left(3 \times 10^{19} \mathrm{eV}\right)$, and a Galactic component $(G)$ which has a slope of about -3 (i.e. flat in Fig. 1) well before the knee, with the spectrum steepening at higher energies, because of the propagation characteristics, to a roughly Gaussian form beyond the ankle. The reason for adopting the specific mass composition shown in Fig. 1 will be considered later after an analysis of the magnetic properties of the interstellar medium and the ensuing effect on the CR spectrum.

The slope of the EG component (about -2.4) is not welldefined because of the short range of energy before the GZK reduction. It differs from the $-2.59 \pm 0.02$ quoted by the PAO group (Schussler et al., 2010) in their "broken-power law fit" to the overall spectrum because such a fit would only be true if all the $\mathrm{CR}$ above a few times $10^{17} \mathrm{eV}$ were Extragalactic.

\subsection{Regular Galactic magnetic field}

There are a number of models of the regular Galactic magnetic fields. We have tested some of them (Wibig and Wolfendale, 2010) and found that differences concerning the subject of the present paper are not substantial. In any event, there are still many uncertainties in the magnetic properties of the interstellar medium which affect the propagation of

Published by Copernicus Publications on behalf of the Arbeitsgemeinschaft Extraterrestrische Forschung e.V. 


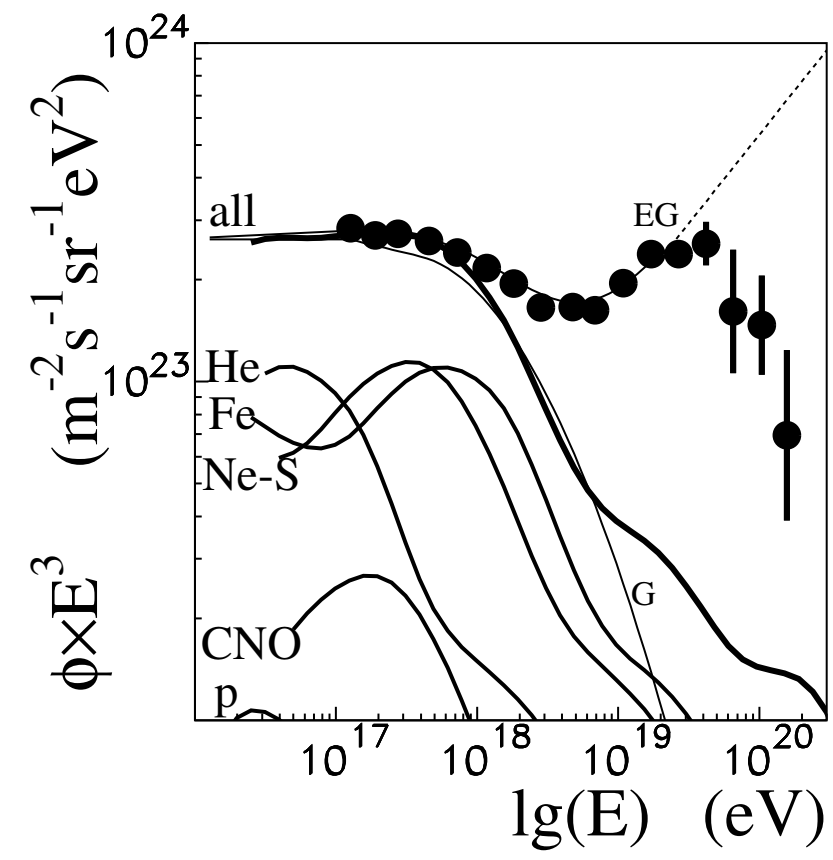

Fig. 1. The UHECR flux and its $G$ and EG components shown with the comparison of simulated Galactic component cosmic rays from sources distributed uniformly in the Galactic disk (the "undulations" and vertical cut-offs are not meaningful).

CR of all energies. Our conclusions are not sensitive to the actual field model adopted. For the present calculations we used the model of Alvarez-Muñiz and Stanev (2006).

The regular Galactic field in the disk is assumed to have a two-arm logarithmic bi-symmetric spiral with radial reversal form. The local regular magnetic field in the vicinity of the Solar System is assumed to be $\sim 1.5 \mu \mathrm{G}$ and the pitch angle is $p=-10^{\circ}$. The field decreases with Galactocentric distance as $1 / r$ and it is zero for $r>20 \mathrm{kpc}$. In the region around the Galactic Center the field is highly uncertain, and it is assumed that it is constant and equal to its value at $r=4 \mathrm{kpc}$. The spiral field strengths above and below the Galactic Plane are taken to decrease exponentially with two scale heights. The additional dipole field is assumed to be toroidal and its strength decreases with Galactocentric distance as $1 / r^{3}$ and is $0.3 \mu \mathrm{G}$ in the vicinity of the Solar System directed toward North.

\subsection{Random component of the Galactic magnetic field}

The random component of the magnetic field is important at all energies. Below several PeV sub-parsec field irregularities give rise to the well-known mean CR lifetime against escape proportional to $E^{-0.5}$. At higher energies, larger scale irregularities dominate, together with the regular field. Here we adopt the Fourier modes and their power spectrum is given by

$B(x)=\int \frac{d^{3} k}{(2 \pi)^{3}} B(k) e^{i(k \cdot x+\phi(k))}$,

in the expression, $k=2 \pi / L$, where $L$ is the magnetic field turbulence scale; in that work the upper and lower limits of the turbulence scales were taken as 100 and $1 \mathrm{pc}$.

The spectral shape predicted is governed by the form of $B(x)$, the adopted injection spectrum and the limits to $L$. The important energy at which steepening starts to become significant in Fig. 1 gives a rigidity of about $30 \mathrm{PV}$, i.e. for example, $6 \times 10^{16} \mathrm{eV}$ for Helium. This can be understood in terms of prompt leakage from the Galaxy volume, where the assumed random magnetic fields could not ensure the diffusion conditions.

More details are given in Wibig and Wolfendale (2010).

\subsection{Results of the Galactic CR propagation model}

The model assumed a "standard" mixed composition, as derived by us from the KASCADE measurements of Apel et al. $(2009 a, b)$. It was adjusted slightly in energy scale to the recent "world average" energy spectrum of UHECR and the final spectrum decomposed to $\mathrm{G}$ and $\mathrm{EG}$ components is shown in the Fig. 1. The random component average strength was found to be of about $3 \mu \mathrm{G}-$ a reasonable value.

\section{Composition}

To make conclusions about the composition of both components we have introduced 8 degrees of freedom describing fractions of each mass group (protons, $\mathrm{He}, \mathrm{CNO}, \mathrm{Ne}-\mathrm{S}, \mathrm{Fe}$ ) separately in the G- and EG fluxes.

The cut-off of the Galactic component was assumed to be $Z$ dependent as is expected when the termination of the $\mathrm{G}$ energy spectrum is related to the confinement of CR by the Galactic magnetic fields. This assumption was tested with different regular magnetic field structures assuming commonly acceptable values of the field, regular, as well as random. Lines in Fig. 1 show predictions of the Galactic flux (with typical, low energy mass composition) observed in the vicinity of the Solar System assuming a uniform distribution of CR sources in the Galactic disk. As is seen, our assumption about the Gaussian shape of the cut-off works well in the case of real Galactic magnetic fields.

\section{The Depth of Shower Maximum}

With the simple analytical form of the fluxes we can apply the fitting procedure to the PAO data on $x_{\max }$. The data can be utilize only by the comparison with predictions of the shower development simulation code. We have used the results published together with the data by the PAO group for the average $x_{\max }$ as well as the $x_{\max }$ spread (RMS). The calculated 


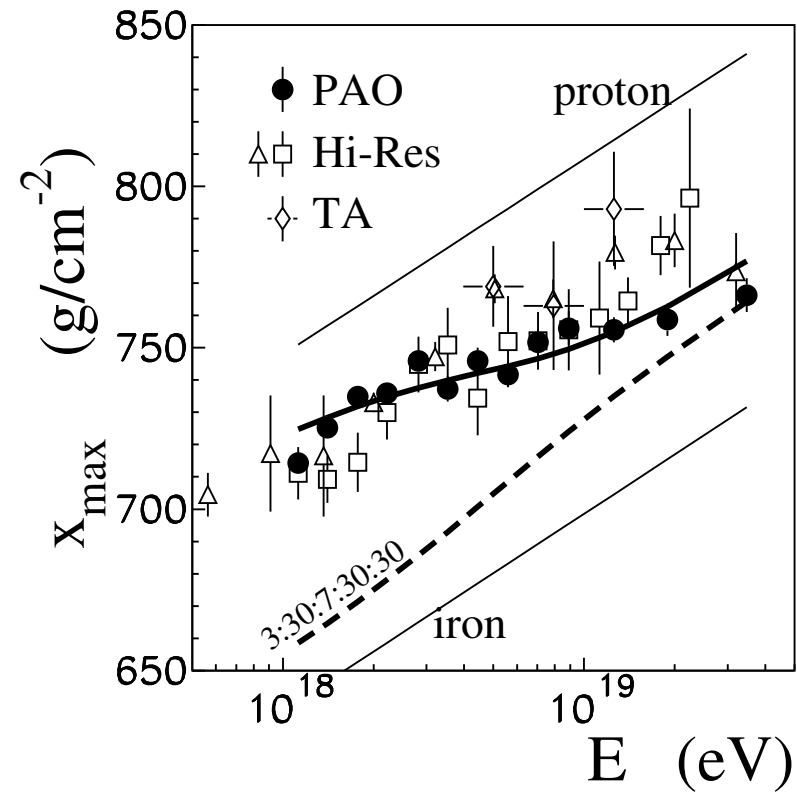

Fig. 2. Average position of the shower maximum as a function of primary particle energy. Thick solid line shows results of the composition fit, the dashed - results of the heavy composition model (p:He:CNO:Ne-S:Fe = 3\%:30\%:7\%:30\%:30\%).

results were given for proton and iron primaries only. For intermediate masses we applied the simple $\ln (A)$ dependence. The HiRes $x_{\text {max }}$ data have been included for completeness.

Results of fitting 8 parameters to the average $x_{\max }$ and its RMS data are shown in Figs. 2 and 3. The fractions of components and eventual energy spectrum are shown in Figs. 4 and 5 .

It is immediately apparent that the mean mass $(\langle\ln A\rangle) \mathrm{ob}-$ served by PAO is inconsistent with that from Fig. 1. The latter has a much higher mean mass than PAO, as can be seen in Fig. 2. In order to provide the fall-off in the G-component at about $5 \times 10^{17} \mathrm{eV}$ with the field model adopted many heavy nuclei are required. The possible reasons are given in Sect. 5 .

\section{Discussion and Conclusions}

Although the status of the energy spectrum itself is good, with the PAO results being consistent with the rest of the World's data, it is evident that there are problems with the mass composition. Specifically, there is the fact that from Fig. 1 we expect at $10^{18} \mathrm{eV}$ a preponderence of heavy nuclei (specifically giving $\langle\ln A\rangle=2.1$ for which the depth of maximum should be about $675 \mathrm{~g} \mathrm{~cm}^{-2}$ ) wheras PAO give $\langle\ln A\rangle=1$ (Fig. 2) and $x_{\max }=715 \mathrm{~g} \mathrm{~cm}^{-2}$. There are a number of possible explanations, as follows:

a. The PAO results are correct but the problem lies with the adopted magnetic field. The knee position in energy is proportional to the magnitude of the mean ran-

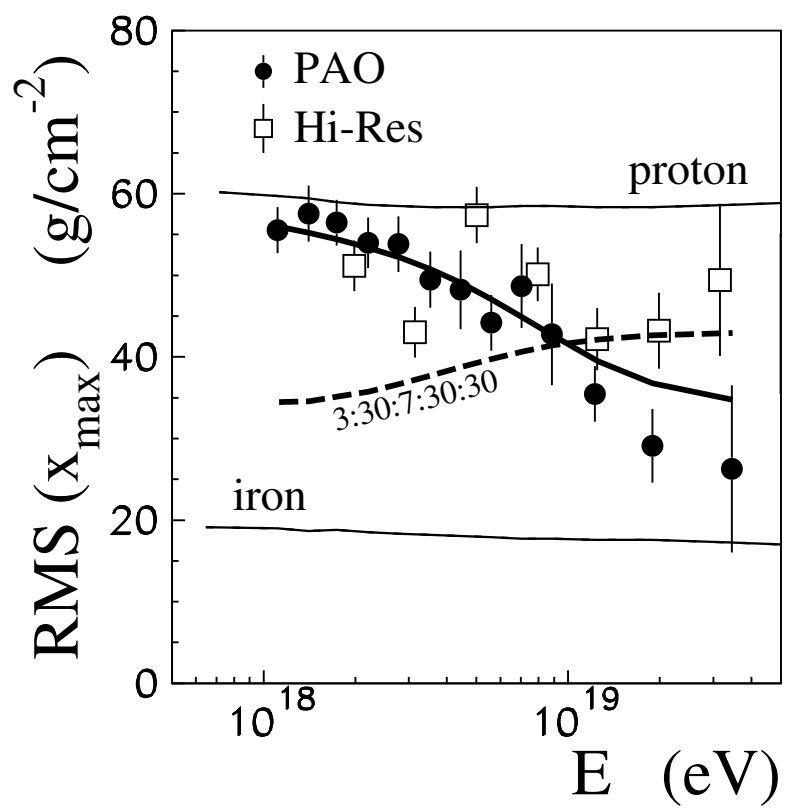

Fig. 3. RMS of the $x_{\max }$ from the composition fit (thick solid line) and heavy composition (dashed line) compared with data.

dom field. However an increase of a factor 10 is rquired and this is not physical. For one thing it is not evident where the magnetic field energy would come from; at present there is equipartition of field energy and total CR energy and this equipartition would then fail. Some alleviation would arise if there were more magnetic energy in the long scale irregularities. A good feature of the PAO results is, however, that there is consistency between Figs. 2 and 3, in which the particles are "light".

b. The PAO results are incorrect. This suggestion is not put forward lightly but is made because of the fact that most other measurements give significantly smaller $x_{\max }$ values and, correspondingly, higher $\langle\ln A\rangle$ values. Inspection of the World summary by Pierog et al. (2009) shows that most other sets of data are clustered round $x_{\max }=675 \mathrm{~g} \mathrm{~cm}^{-2}$ at $10^{18} \mathrm{eV}$. It must be said however that the spread in $x_{\max }$ values is rather large.

By $10^{19} \mathrm{eV}$ the PAO and the rest of the World's $x_{\max }$ values have come together with $x_{\max }$ about $650 \mathrm{~g} \mathrm{~cm}^{-2}$ and $\langle\ln A\rangle=1.6$. This can be compared with the $\langle\ln A\rangle=2.5$ from Fig. 1 for Galactic particles alone, but the difference can be rectified, partly at least, by postulating that the EG particles are very massive.

c. It is not impossible that the problem with the $\langle\ln A\rangle$ values is connected with the nuclear physics model used to convert $x_{\max }$ to $\langle\ln A\rangle$. Although this would not account for the discrepancies in the experimental values from the different experiments, the PAO vaues of $x_{\max }$ could be correct yet the particles could be heavy. One 


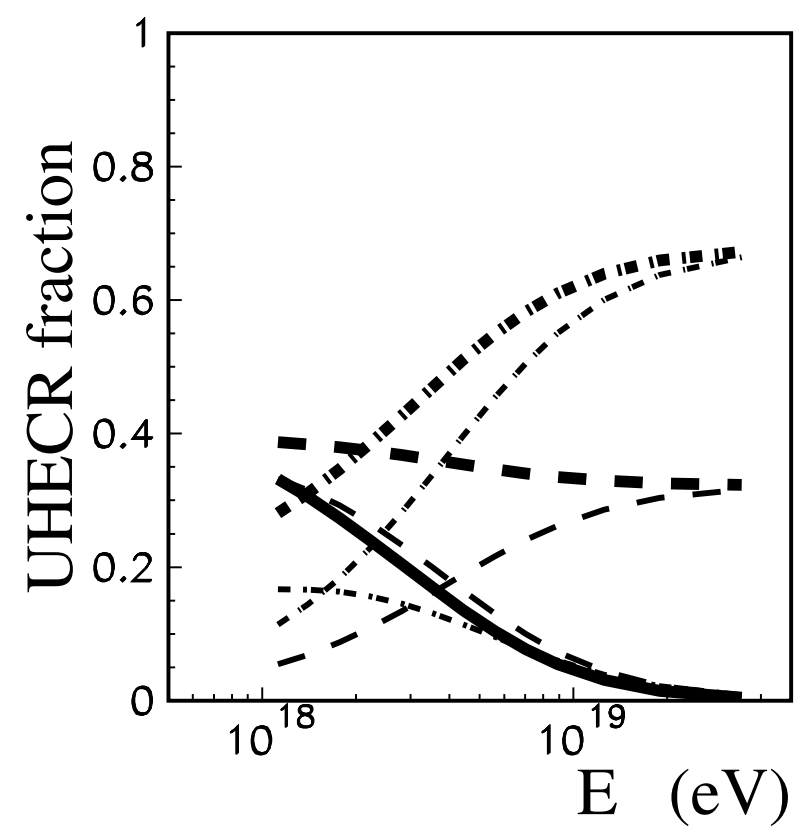

Fig. 4. Fractions of the different mass groups in the UHECR flux for the best composition fit. Solid line shows the proton contribution, dashed lines - helium and dot-dashed - CNO group. Thin lines show $\mathrm{G}$ and EG contributions separately while thick lines are for the sum of them.

of us (Wibig, 2010) has considered a "Scale-breaking models" which would give this result.

The conclusion is, in our view, that there are serious problems remaining in the search for the mass composition of $\mathrm{CR}$ at energies above $10^{17} \mathrm{eV}$.

Edited by: R. Vainio

Reviewed by: two anonymous referees

\section{References}

Abbasi, R. U. et al. (Hi-Res Collaboration): Indications of ProtonDominated Cosmic-Ray Composition above 1.6 EeV, Phys. Rev. Lett., 104, 161101, 2010.

Abraham, J. et al. (Pierre Auger Collaboration): Measurement of the Depth of Maximum of Extensive Air Showers above $10^{18} \mathrm{eV}$, Phys. Rev. Lett., 104, 091101, 2010.

Alvarez-Muñiz, J. and Stanev, T.: The Large Scale Structure of the Galactic Magnetic Field and High Energy Cosmic Ray Anisotropy, J. Phys. Conf. Ser., 47, 126-131, 2006.

Apel, W. D. et al. (KASCADE-Grande Coll.): in: Proc. 31st ICRC, Łódź, Poland, 2009a.

Apel, W. D. et al. (KASCADE-Grande Coll.): Report FZKA 7516, Forschungszentrum Karlsruhe, 2009b.

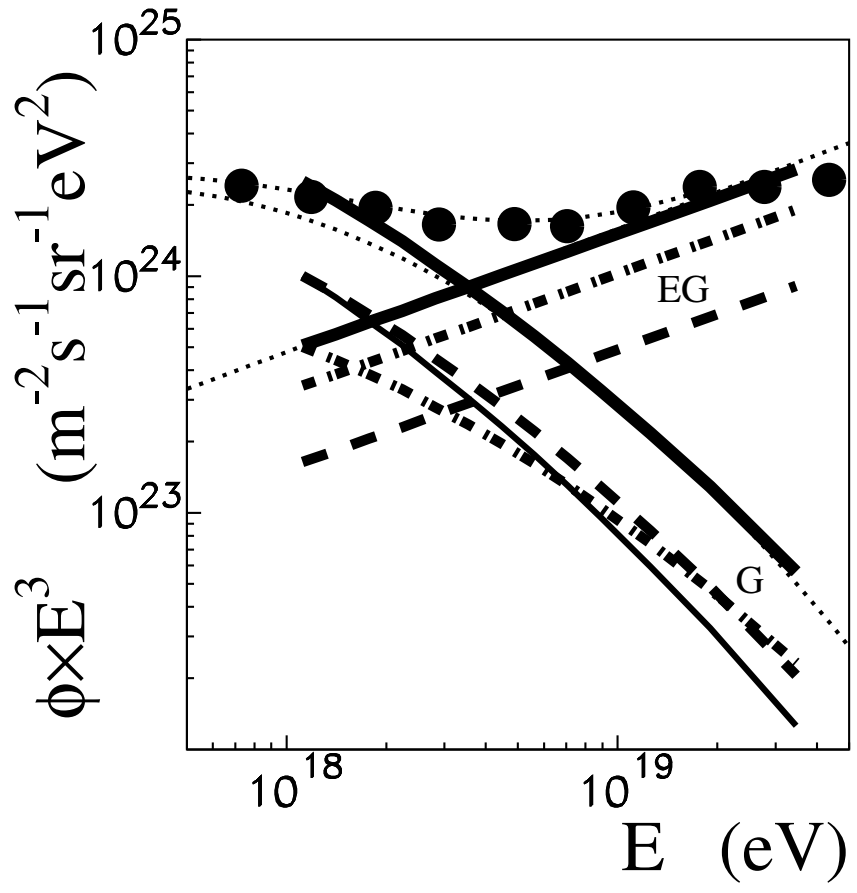

Fig. 5. Final UHECR spectrum fit shown separately for $G$ and EG mass components. Only non negligible components are shown. Thin solid line shows the proton contribution, dashed lines - helium and dot-dashed - CNO group. Thick solid lines are for the G and EG components in total to be compared with the model prediction given by two dotted lines for each component and their sum - model UHECR spectrum.

Berezinsky, V. S., Grigorieva, S. I., and Hnatyk, B. I.: Extragalactic UHE proton spectrum and prediction for iron-nuclei flux at $10^{8}-$ $10^{9} \mathrm{GeV}$, Astropart. Phys., 21, 617-625, 2004.

Pierog, T., Engel, R., and Heck, D.: 3-D Air Shower Simulations Using CONEX in CORSIKA, in: Proc. 31st ICRC, Łódź, Poland, 129-132, 2009.

Schüssler, F. for the Pierre Auger Collaboration: Measurement of the cosmic ray energy spectrum above $10^{18} \mathrm{eV}$ using the Pierre Auger Observatory, in: Proc. XXXI ICRC Łódź, Poland, 2010.

Sokolsky, P. and Thomson, G. B.: Highest energy cosmic-rays and results from the HiRes experiment, J. Phys. G, 34, 401, 2007.

Szabelski, J., Wibig, T., and Wolfendale, A. W.: Cosmic rays of the highest energies: the case for extragalactic heavy nuclei, Astropart. Phys., 17, 125-131, 2002.

Wibig, T.: Ultra high-energy cosmic ray proton interactions, Phys. Let B, 678, 60-64, 2009.

Wibig, T. and Wolfendale, A. W.: At what particle energy do extragalactic cosmic rays start to predominate?, J. Phys. G, 31, 255264, 2005.

Wibig, T. and Wolfendale, A. W.: The spectrum of Galactic Cosmic Rays of the highest energies, in: Proc. XXXI ICRC Łódź, Poland, 2010. 